\title{
Downregulation of Bcl-2, FLIP or IAPs (XIAP and survivin) by siRNAs sensitizes resistant melanoma cells to Apo2L/TRAIL-induced apoptosis
}

\author{
M Chawla-Sarkar ${ }^{1}$, SI Bae ${ }^{1}$, FJ Reu ${ }^{1}$, BS Jacobs ${ }^{1}$, DJ Lindner ${ }^{1}$ \\ and EC Borden ${ }^{*, 1}$ \\ ${ }^{1}$ Center for Drug Discovery and Development, Taussig Cancer Center, \\ Cleveland Clinic Foundation, Cleveland, OH, USA \\ * Corresponding author: EC Borden, Taussig Cancer Center, 9500 Euclid Ave. \\ R-40, Cleveland, OH 44195, USA. Tel: + 216444 8183; Fax: 216636 2498; \\ E-mail: bordene@cc.ccf.org
}

Received 26.8.03; revised 31.9.03; accepted 02.12.03; published online 30.4.04 Edited by Dr T Ferguson

\section{Abstract}

Melanoma cells are relatively resistant to Apo2L/TRAIL (TNFrelated apoptosis-inducing ligand). We postulated that resistance might result from higher expression of inhibitors of apoptosis including Bcl-2, FLIP (FLICE-like inhibitory protein) or IAPs such as XIAP (X-linked inhibitor of apoptosis) or survivin. Compared to scrambled or mismatch controls, targeting individual inhibitors with siRNA (si-Bcl-2, si-XIAP, si-FLIP or si-Surv), followed by Apo2L/TRAIL resulted in marked increase in apoptosis in melanoma cells. Compared to Bcl-2 or FLIP, siRNAs against XIAP and survivin were most potent in sensitizing melanoma cells. A similar substantial increase in apoptosis was seen in renal carcinoma cells (SKRC-45, Caki-2), following the inhibition of either XIAP or survivin by siRNAs. Apo2L/TRAIL treatment in IAP-targeted cells resulted in cleavage of Bid, activation of caspase- 9 and cleavage of PARP (poly ADP-ribose polymerase). Thus, Apo2L/TRAIL resistance can be overcome by interfering with expression of inhibitors of apoptosis regulating both extrinsic (death receptor) or intrinsic (mitochondrial) pathways of apoptosis in melanoma cells.

Cell Death and Differentiation (2004) 11, 915-923.

doi:10.1038/sj.cdd.4401416

Published online 30 April 2004

Keywords: siRNA; XIAP; Bcl-2; FLIP; Survivin; Apo2L/TRAIL

Abbreviations: IAP, inhibitor of apoptosis; XIAP, X-linked inhibitor of apoptosis; FLIP, flice-like inhibitory protein; siRNA, small interfering ribonucleic acid; Apo2L/TRAIL, TNF-related apoptosis-inducing ligand; si-Scr, random scrambled siRNA; IFN, interferon

\section{Introduction}

Apoptosis plays a critical role in differentiation, in elimination of cells that sustain genetic damage or undergo uncontrolled cellular proliferation. ${ }^{1-3}$ With recent advances in molecular genetics, it has become evident that malignant cells commonly have defects in cell death control and apoptosis. ${ }^{1-3}$ Inhibition of apoptosis can lead to tumorigenesis and resistance to therapy. ${ }^{4}$ The antiapoptotic proteins, Bcl- $2,{ }^{5-7}$ Flice-inhibitory protein or FLIP ${ }^{8,9}$ and inhibitors of apoptosis (IAPs) such as X-linked inhibitor of apoptosis (XIAP), c-IAP and survivin, are elevated in a range of human cancers including melanomas, prostate carcinoma and gliomas. ${ }^{4,10}$ Thus, targeting IAPs represents a promising strategy for a wide spectrum of malignancies. ${ }^{4,10,11}$

Although the precise mechanism by which they exert their antiapoptotic effects is unclear, the cellular stoichometry of the antiapoptotic Bcl-2 family members compared with their apoptotic homologues (Bax) often defines the susceptibility of cells to a death signal. ${ }^{5-7}$ FLIP primarily blocks apoptosis induced by death receptors such as Fas, TRAIL-R and TNF$R 1$. High levels of FLIP ${ }_{L}$ have been reported in melanoma and metastatic cutaneous melanoma lesions from human patients. $^{8,9}$ IAPs negatively regulate apoptosis by inhibiting caspase activity. ${ }^{11,12}$ Caspases form a proteolytic network that is responsible for cleavage of other proteins, leading to morphological manifestations of apoptosis. ${ }^{2,5}$ IAPs regulate apoptosis both by preventing the action of central execution phase through direct inhibition of the effector caspase-3 and/ or caspase-7 and in addition, prevent initiation of the intrinsic caspase cascade by directly inhibiting the apical caspase- 9 . However, IAPs also play a role in cell-cycle regulation, ubiquitination and proteosome-mediated protein degradation. ${ }^{11-13}$

Apo2L/TRAIL is a transmembrane protein that shares homology in its extracellular domains with other members of the tumor necrosis factor (TNF) family. ${ }^{14,15}$ Induction of Apo2L/TRAIL in response to IFNs correlated inversely with resistance to IFN-induced apoptosis in melanoma cell lines. ${ }^{16}$ However, most of the melanoma cell lines were relatively resistant to recombinant Apo2L/TRAIL protein as a single agent. ${ }^{17-19}$ Overexpression of XIAP and FLIP has been implicated to confer Apo2L/TRAIL resistance in melanoma and other tumors. ${ }^{18,19}$ Sensitization of melanoma cells to Apo2L/TRAIL-induced apoptosis by IFN- $\beta$ pretreatment correlated with the cleavage of XIAP. ${ }^{17}$

To test whether the death-inducing potential of Apo2L/ TRAIL could be enhanced by downregulation of inhibitors of apoptosis, Bcl-2, XIAP, FLIP, or survivin, suppression of gene expression by small (synthetic 21-base duplex RNA) interfering RNA (siRNA), ${ }^{20-22}$ was utilized to inhibit their expression in melanoma and renal carcinoma cells. The results show that these inhibitors play an important role in mediating resistance to apoptotic effects of the cytokines Apo2L/ TRAIL and TNF- $\alpha$ but this resistance can be reversed significantly by downregulation of their expression. 


\section{Results}

Downregulation of Bcl-2, FLIP, XIAP and survivin protein expression utilizing 21-mer synthetic siRNAs in melanoma cells

Melanoma cells (A375) were transfected either with lipofectamine alone, a control siRNA (a random scrambled sequence; si-Scr), or with gene-specific siRNAs $(20-40 \mathrm{nM})$ targeted against $\mathrm{Bcl}-2$ (si-Bcl-2), XIAP (si-XIAP), survivin (si-Surv) or FLIP (si-FLIP). Cells were analyzed at 48 and $72 \mathrm{~h}$ posttransfection for protein expression by immunoblotting. All siRNAs utilized in this study downregulated protein expression of respective genes by $50-90 \%$ (Figure 1a). To address the issue of gene specificity ${ }^{21}$ or nonspecific off-target effects $^{23}$ of siRNAs used in the study, mismatch (2-4 nt) siRNAs were designed against si-Bcl-2 (si-Bcl-2 MM) and siXIAP (si-XIAP MM). A375 cells were transfected with $20 \mathrm{nM}$ of either mismatch siRNA or siRNAs against Bcl-2 or XIAP. Lipofectamine control as well as scrambled siRNA were also used as internal controls. Both si-Bcl-2 and si-XIAP inhibited protein expression (50-80\%). However, similar to si-Scr, mismatch siRNA had no significant effect on expression of either XIAP or Bcl-2 expression (Figure 1b,c). si-XIAP downregulated XIAP but no such effect was observed on expression of either Bcl-2 or survivin (Figure 1b). Similarly, si$\mathrm{Bcl}-2$ was specific for $\mathrm{Bcl}-2$ as no downregulation of $\mathrm{Bax}$, Bad or XIAP proteins was observed (Figure 1c). At higher concentrations of si-XIAP ( $>60-100 \mathrm{nM})$, nonspecific downregulation (30-50\%) of survivin was observed, which correlated with increased si-RNA-associated cytotoxicity in cells (data not shown).

Since siRNA has been shown to result in Interferon (IFN) gene induction in some cells, ${ }^{24,25}$ A375 cells transfected with chemically synthesized $21 \mathrm{nt}$ siRNAs were also analyzed for two different IFN-stimulated genes (ISGs), signal transducer and activator of transcription 1 (STAT-1) and ISG15 (p15). Both STAT-1 and ISG15 are induced very strongly ( $>10$-fold) in most cells in response to IFNs or viral infection. ${ }^{26}$ Compared to controls, the XIAP-specific siRNAs utilized in this study did not induce STAT-1 or ISG15 protein expression in A375 cells (Figure 1d). Treatment with IFN- $\alpha 2(25 \mathrm{U} / \mathrm{ml})$ for $16 \mathrm{~h}$ resulted in strong induction of both STAT-1 and ISG15 in A375 cells. Induction of two other ISGs (UBE2L6 and USP18) was analyzed in $A 375$ cells following transfections with siXIAP or lipofectamine alone by quantitative PCR (ABI 7700, Applied Biosystems, Foster City, CA, USA). Compared to wild-type cells (expression $\approx 1$ ), no induction of transcripts of USP18 and UBE2L6 was observed in lipofectamine (1.006 and 1.02)- or si-XIAP (1.07 and 1.09)-transfected cells. Thus at given concentrations, siRNAs utilized in this study were selective, specific and relatively nontoxic.

\section{Inhibition of IAPs reverses Apo2L/ TRAIL resistance in melanoma cells}

In our previous study, ${ }^{17}$ apoptotic effects of Apo2L/TRAIL were assessed in melanoma cells. No significant apoptosis ( $<10 \%)$, as measured by Annexin V/propidium iodide $(\mathrm{PI})$ staining, was observed following $25-200 \mathrm{ng} / \mathrm{ml}(24-48 \mathrm{~h})$

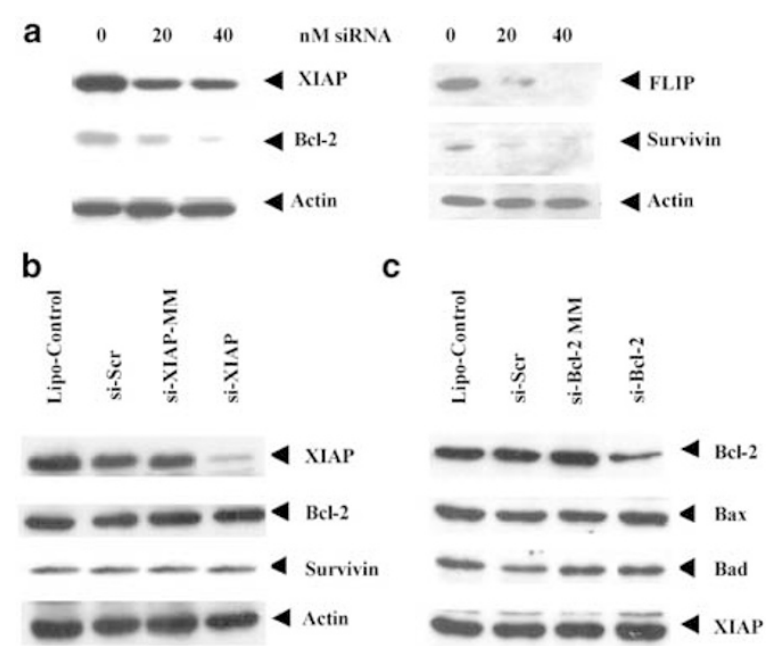

d

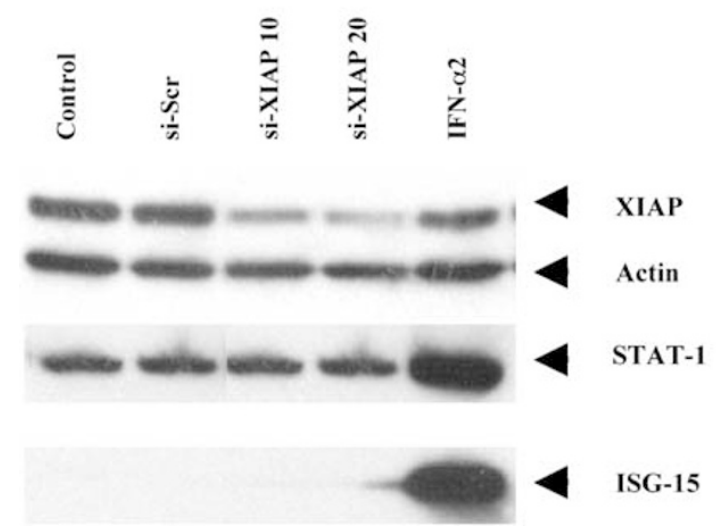

Figure 1 Si-RNAs specific for IAPs potently suppress gene expression in A375 melanoma cells. (a) Gene-specific siRNAs and the control siRNAs (20-40 nM) were added to the media using lipophilic transfection-enhancing reagent (Lipofectamine 2000, Invitrogen Inc.). Cells were harvested after $48 \mathrm{~h}$ and immunoblot analyses were performed using Bcl2, XIAP, FLIP and survivin specific antibodies. The blots were reprobed with antibody against actin to confirm equal protein loading. Si-RNAs effectively inhibited expression of specific genes by $60-90 \%$ (three independent experiments). (b) Whole cell extracts prepared from A375 cells transfected with si-Scr, si-XIAP MM and si-XIAP siRNAs were immunoblotted with antibodies against XIAP, Bcl-2 and survivin. Control siRNAs, si-XIAP MM and si-Scr had no effect; however, Si-XIAP effectively inhibited XIAP expression. No downregulation of $\mathrm{Bcl}-2$ or survivin expression was observed. (c) Whole cell extracts prepared from A375 cells transfected with si-Scr, si-Bcl-2 MM and si-Bcl-2 siRNAs were immunoblotted with antibodies against Bcl-2, Bax, Bad and XIAP. Control siRNAs, si-Bcl-2 MM and si-Scr had no effect; however, Si-Bcl-2 effectively inhibited (50-60\%) Bcl-2 expression. No effects were observed on expression of Bax, Bad or XIAP proteins. (d) A375 melanoma cells were transfected with si-XIAP. After $48 \mathrm{~h}$, cells were harvested and immunoblot analysis was performed to confirm downregulation of XIAP expression. Blots were reprobed with antibodies against two IFN-stimulated genes, STAT-1 and ISG-15. No induction of STAT1 or ISG-15 was observed following transfection with si -XIAP. Cells treated with IFN- $\alpha 2$ (25U/ $\mathrm{ml}$ ) for $16 \mathrm{~h}$ showed induction of both STAT1 and ISG15

Apo2L/TRAIL. Since treatment with Apo2L/TRAIL resulted in activation of caspase cascade, but no apoptosis, ${ }^{17}$ a possible role of downstream inhibitors of apoptosis such as $\mathrm{Bcl}-2$ and XIAP that bind to FADD or other proteins in caspase pathway has been postulated. ${ }^{17-19}$ 
To determine the role of various inhibitors of apoptosis in mediating resistance to Apo2L/TRAIL, a melanoma cell line (A375), defective in endogenous Apo2L/TRAIL gene induction, was chosen. ${ }^{16}$ To determine whether si-XIAP might augment apoptosis, cells were transfected either with lipofectamine alone, control siRNAs (si-Scr, si-XIAP MM), or with si-XIAP (Figure 2a). Although some nonspecific apoptosis occurred in controls (15-23\%), marked augmentation in apoptosis occurred only with si-XIAP (Figure 2a). Similar effects were observed when cells were transfected with si-Bcl2 , si-FLIP or si-Surv, followed by treatment with either TNF- $\alpha$ $(50 \mathrm{ng} / \mathrm{ml})$ or Apo2L/TRAIL $(100 \mathrm{ng} / \mathrm{ml})$ for $12-16 \mathrm{~h}$. Apoptotic cell death was assessed by both Annexin V/PI staining and TUNEL staining followed by bivariate FACS analysis (Figure $2 \mathrm{~b}, \mathrm{c})$. No significant apoptosis $(<10-12 \%)$ was observed in cells transfected with lipofectamine reagent (Figure $2 a, b)$. However, when cells transfected with si-Bcl-2, si-XIAP, siSurv or si-FLIP were treated with Apo2L/TRAIL, a significant increase (35-70\%) in TUNEL positivity resulted (Figure $2 b$ ). si-XIAP was most potent in sensitizing melanoma cells to Apo2L/TRAIL-induced apoptosis (Figure 2a,b). Control siRNAs (si-Scr, si-Bcl-2 MM or si-XIAP MM) and Apo2L/ TRAIL combination resulted in $15-20 \%$ apoptotic cells. Compared to untreated controls, TNF- $\alpha$ resulted in two-fold increase in apoptosis (15-25\%). Similar results were obtained with Annexin V/PI staining. The lipid-based transfection reagent resulted in increased background Annexin V positivity $(\approx 20$ $\%$ ) compared to controls (Figure 2c).

To confirm that our observations were common to other melanomas, WM9, WM35, MUM2C and C918 cell lines were transfected with specific siRNAs and treated with Apo2L/ TRAIL as above. Apo2L/TRAIL (16 h) alone had no significant effect on TUNEL staining (5-10\%). However, siRNAs against different inhibitors sensitized WM9, MUM2C and C918 cells to Apo2L/TRAIL-induced apoptosis. As with A375, siRNA against XIAP was most potent in rendering melanoma cells TRAIL sensitive (Figure 3). Of the five melanoma cell lines, only WM35 were relatively resistant to Apo2L/TRAILmediated apoptosis ( $\approx 28-35 \%$ apoptosis in si-XIAP-transfected cells). Even though the percent apoptosis compared to other cell lines was low, it was still significant compared to controls $(P=0.0182)$. Resistance in these cells could be due to either lower transfection efficiency (only 30-50\% downregulation of XIAP was observed in three different experiments) or defects in Apo2L/TRAIL signaling pathway. These cells were also resistant to Apo2L/TRAIL-induced apoptosis in combination with other therapeutics like IFN- $\beta$ or nitrosylcobolamin. ${ }^{17,27}$ In the renal cell carcinoma cells, SKRC-45 (Figure 3) and Caki-2 (data not shown), both si-XIAP and si-Surv were potent in sensitizing cells to apoptosis (50-80\%). No significant apoptosis was observed following siRNA and TNF- $\alpha$ combination in either melanoma or renal cell carcinoma
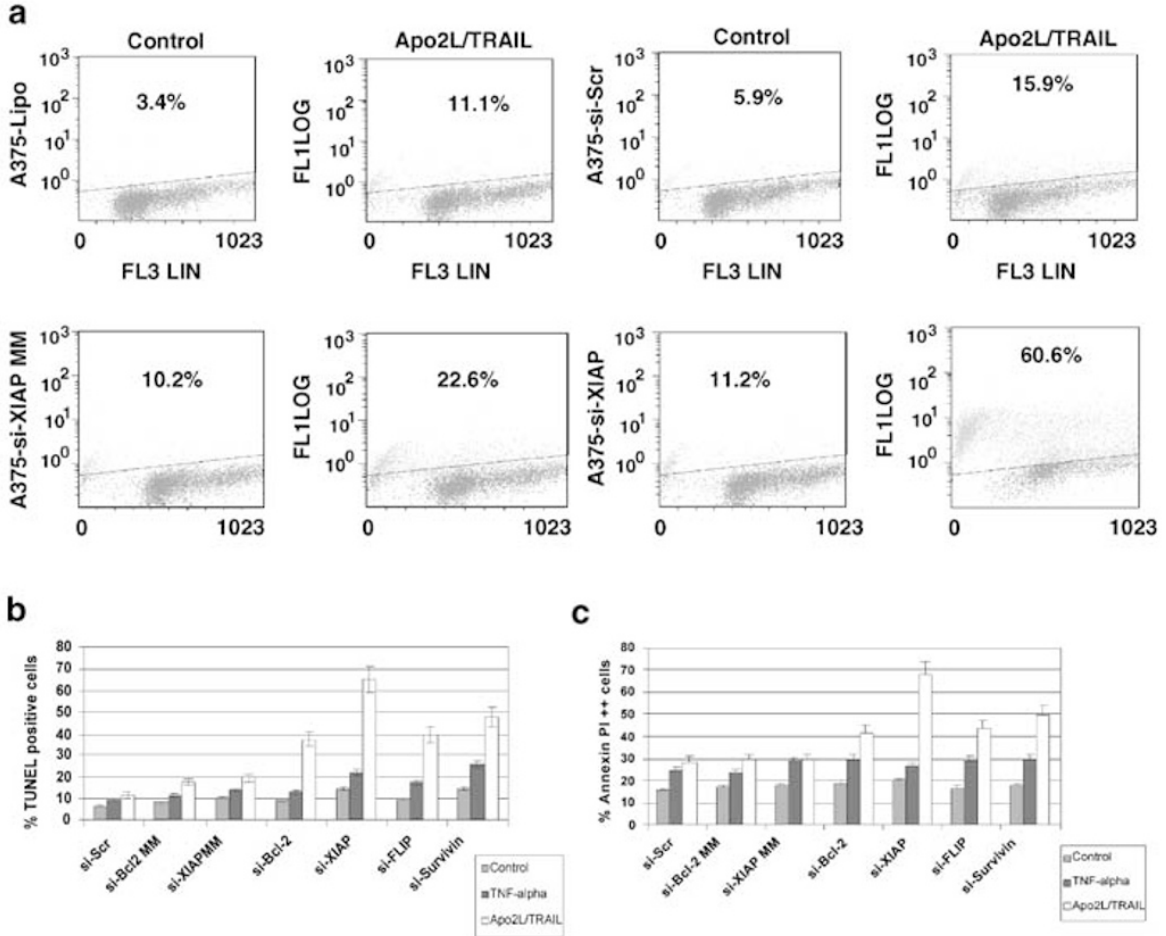

Figure 2 Inhibition of IAPs sensitizes A375 melanoma cells to TRAIL/Apo2L-induced apoptosis. (a) A375 cells transfected with Lipofectamine 2000 alone, control siScr, si-XIAP MM or with si-XIAP $(20 \mathrm{nM})$ were treated with TRAIL/Apo2L $(100 \mathrm{ng} / \mathrm{ml})$ for $16 \mathrm{~h}$. DNA fragmentation was detected by TUNEL analysis. Cells were fixed, labeled with Br-dUTP by the enzyme TdT and then stained with FITC-labeled anti-BrdU mAb. The percentage of FITC-positive cells was assessed by FACS analysis. Representative histograms of cells, transfected with si-Scr, si-XIAP-MM and si-XIAP followed by Apo2L/TRAIL are shown from one experiment. (b) A375 cells transfected with IAP-specific siRNAs or with control siRNA (si-Scr, si-Bcl2-MM, si-XIAP MM) were treated with TNF- $\alpha$ (50 ng/ml) or TRAIL/Apo2L (100 ng/ml) for $16 \mathrm{~h}$, then harvested and analyzed for apoptosis by TUNEL analysis. Graph represents mean of three independent experiments. (c) A375 cells treated with TNF- $\alpha$ and Apo2L/ TRAIL (12 h) were stained with Annexin V/PI and subjected to bivariate FACS analysis. The percentage represents sum of Annexin V-positive and Annexin + PI doublepositive cells 

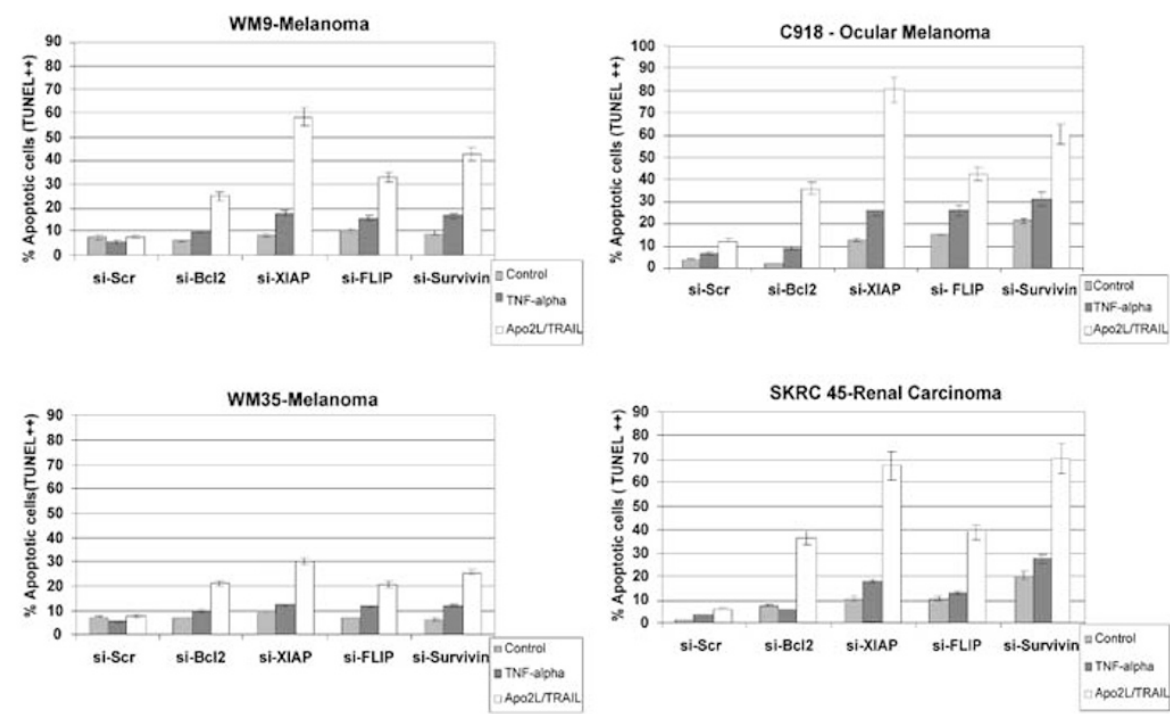

Figure 3 Reversal of Apo2L/TRAIL resistance by si-RNAs against IAPS in melanoma and renal carcinoma cell lines. DNA fragmentation was detected by TUNEL analysis. Melanoma (WM9, WM35), ocular melanoma (C918) and renal cell carcinoma (SKRC45) cells transfected with IAP-specific siRNAs or with control siRNA, were treated with TNF- $\alpha(50 \mathrm{ng} / \mathrm{ml})$ or TRAIL/Apo2L (100 ng/ml) for $16 \mathrm{~h}$. The cells were fixed, labeled with Br-dUTP and then stained with FITC-labeled anti-BrdU mAb. The percentage of FITC-positive cells was assessed by FACS

cells (Figure 3). Apo2L/TRAIL is a tumor-specific inducer of apoptosis with little or no toxicity towards primary cells. ${ }^{28}$ To assess whether siRNAs against inhibitors of apoptosis would sensitize primary cells to TRAIL-induced apoptosis, the effect of si-XIAP was analyzed in diploid cells, DMN-1 (melanocyte) and human foreskin fibroblasts (HFF). Primary cells expressed lower levels of XIAP compared to A375 melanoma cells. Transfections of DMN-1 or HFF cells with si-XIAP resulted in downregulation of XIAP expression $(\approx 50 \%)$, but did not sensitize them to Apo2L/TRAlL-induced apoptosis $(<15-20 \%)$ as assessed by TUNEL analyses (data not shown).

\section{Apo2L/Trail-induced activation of caspase cascade is amplified by inhibition of IAPs}

To probe the mechanism of apoptotic crosstalk between downregulation of inhibitors of apoptosis and Apo2L/ TRAIL, cleavage of synthetic substrates that indicate caspase activation was assessed. A375 cells were transfected with either control siRNAs or with siRNAs against Bcl-2, XIAP, FLIP and survivin. After $48 \mathrm{~h}$, cells were replated and either left untreated or were treated with Apo2L/ TRAIL for 1, 2 and $4 \mathrm{~h}$. Cell extracts were analyzed for caspase- 3 , caspase- 8 and caspase-9 activity using specific fluorogenic caspase tetrapeptide substrates. Compared to lipofectamine control, siScr, si-Bcl-2 MM or si-XIAP MM transfections had no greater effect on the caspase activity in A375 cells.

Consistent with our previous observation in untransfected A375 cells, ${ }^{17}$ Apo2L/TRAIL (2-4h) resulted in a 5-10-fold increase in caspase-3 enzymatic activity in si-Scr-, si-Bcl-2 MM- or si-XIAP MM-transfected cells. No significant change in caspase-3 activity was observed after $1 \mathrm{~h}$ treatment (Figure 4a). However, compared to controls, si-Bcl-2, siXIAP, si-FLIP and si-Surv followed by Apo2L/TRAIL aug- mented caspase- 3 activity within $1 \mathrm{~h}$ ( $2-5$-fold), which further increased (20-40-fold) in a time (2-4h)-dependent manner (Figure 4a). Maximum increase in activity of caspase-3 was observed until 6-8 $\mathrm{h}$ following Apo2L/TRAIL treatment (data not shown). Consistent with caspase-3 activation, Apo2L/ TRAIL (1-4 h) induced a 1.8-2.5-fold increase in caspase-8 activity in either lipofectamine-control or in cells transfected with either control siRNAs (Figure 4b). However, in cells transfected with si-IAPs, a 3-8-fold increase in caspase-8 activity was observed, $2-4 \mathrm{~h}$ following Apo2L/TRAIL (Figure $4 \mathrm{~b}$ ). si-FLIP was most potent in inducing caspase-8 activation; $\approx 4$-fold activation was observed as early as $1 \mathrm{~h}$. This was further augmented with increasing time (Figure 4b).

Since Apo2L/TRAIL resulted in activation of caspase-3, a block in apoptosis downstream of caspase-3 was postulated. XIAP is a potent IAP that binds and inhibits caspase-9 and caspase-3. ${ }^{29}$ XIAP expression has been associated with Apo2L/TRAIL resistance in melanoma cells. ${ }^{19}$ Thus, the effect of si-IAPs on caspase-9 was also analyzed. In si-Scr-, si-Bcl-2 MM- or si-XIAP MM-transfected cells, Apo2L/TRAIL had no significant effect on caspase-9 activity (Figure 4c). However, in si-Bcl-2-, si-XIAP-, si-Surv- and si-FLIP-transfected cells, treatment with Apo2L/TRAIL resulted in a marked increase (2-6-fold) in caspase-9 activity. Cells transfected with si-XIAP had the greatest increase in caspase-9 activity following Apo2L/TRAIL treatment (Figure 4c). Thus, failure to activate caspase-9 was partly responsible for resistance to TRAILinduced apoptosis.

\section{Apo2L/TRAIL-induced cleavage of Bid: crosstalk between extrinsic and intrinsic pathway}

The effect of si-FLIP-mediated downregulation of FLIP on activation of caspase-8 followed by caspase-3 was expected, since inactivation of this inhibitor would stabilize the signaling 

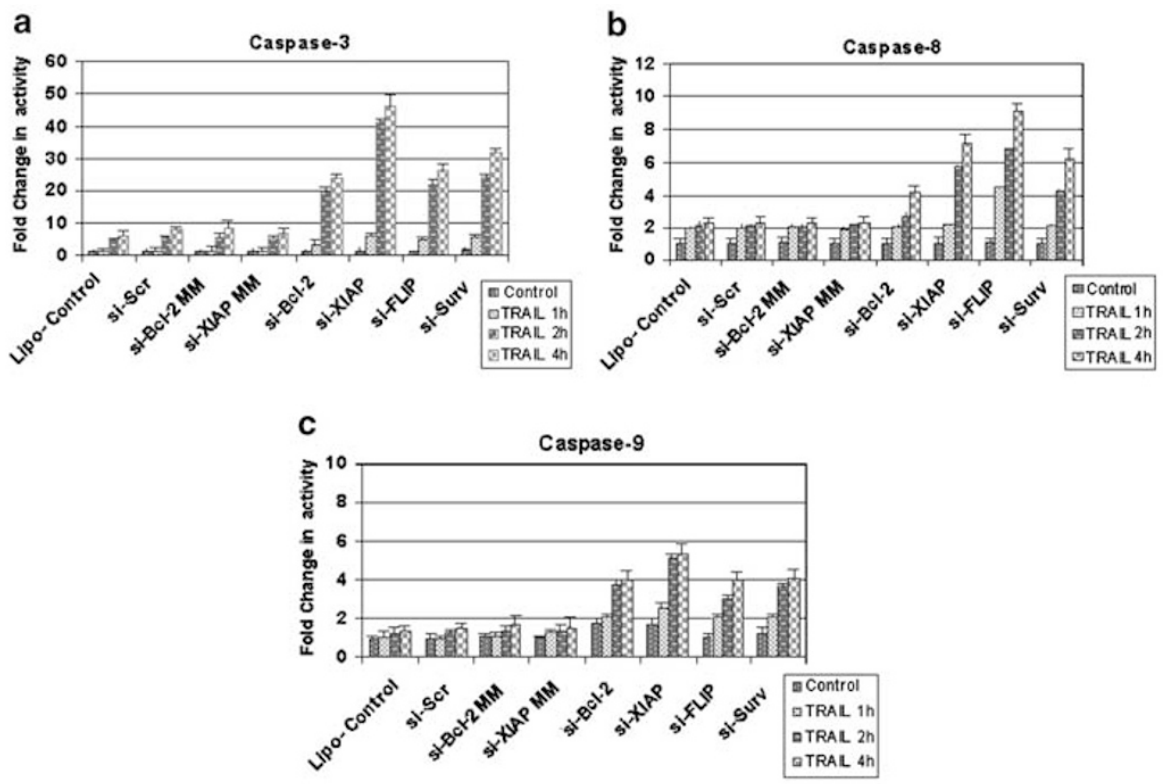

Figure 4 Effect of siRNAs upon caspase enzymatic activity. Caspase-3, caspase-8 and caspase-9 activities were measured using a commercially available ApoAlert assay kit (Clonetech, Palo Alto, CA, USA). siRNA-transfected cells either left untreated or treated with Apo2L/TRAIL (1,2 and $4 \mathrm{~h}$ ) were washed twice with cold PBS and lysed on ice in $50 \mu \mathrm{l}$ cold lysis buffer. Cell lysates were centrifuged at $10000 \times \mathrm{g}$ for $10 \mathrm{~min}$ to remove cellular debris. Cell lysates containing equivalent amount of protein were assayed (in triplicates) for protease activity towards the synthetic fluorogenic substrates of caspase-3 (a), caspase-8 (b) and caspase-9 (c), respectively. Relative fluorescence was measured at $380 / 460 \mathrm{nM}$. Error bars represent mean \pm standard error from three independent experiments

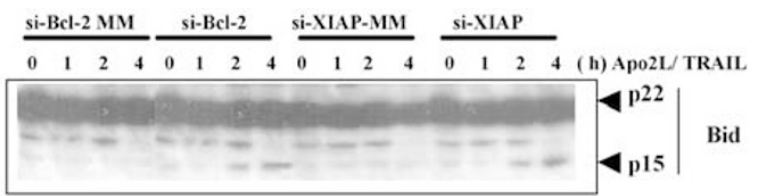

Figure 5 Activation of caspase-cascade by Apo2L/TRAIL in si-Bcl-2- and siXIAP-transfected cells correlated with cleavage of Bid. A375 cells transfected with control siRNAs (si-Bcl-2 MM or si-XIAP MM), si-BCl-2 or si-XIAP were treated with Apo2L/TRAIL (1, 2 and $4 \mathrm{~h}$ ). Total cellular protein was immunoblotted with pAb against Bid, followed by HRP-conjugated anti-rabbit antibody. Bid was cleaved to the $15 \mathrm{kDa}$ proapoptotic fragment by Apo2L/TRAIL (at 2 and $4 \mathrm{~h}$ ) in si-Bcl-2- and si-XIAP-transfected cells

complex. ${ }^{8}$ Similarly, amplification of caspase- 9 and caspase3 activity reflected downregulation of either $\mathrm{Bcl}-2$ or XIAP, since both inhibit caspase-9. However, Apo2L/TRAIL induced a 4-8-fold increase in caspase-8 activity in si-XIAP- or si-Bcl2-transfected cells, which was not expected. To assess a possible interaction connecting the intrinsic (mitochondrial) pathway with the extrinsic (death receptor) pathway, time course analyses were done to analyze cleavage of Bid, a proapoptotic Bcl-2 family member. Activated caspase-8 cleaves caspase-3 as well as Bid. Cleaved or truncated $15 \mathrm{kDa}$ Bid (t-bid) translocates to mitochondria, resulting in release of cytochrome $c$. Released cytochrome $c$ binds to Apaf-1 and procaspase-9 to form an active apoptosome complex. ${ }^{5,6}$ Therefore, immunoblot analysis of Bid was performed in A375 cells transfected either with control siRNAs (si-Bcl2 MM and si-XIAP MM) or with si-Bcl-2 and si-XIAP followed by Apo2L/TRAIL treatment for 1, 2 and $4 \mathrm{~h}$. Consistent with activation of caspase-8 (at 2-4 h) in si-Bcl-2- or si-XIAP-transfected cells, Apo2L/TRAIL induced cleavage of $\mathrm{Bid}$ to a $15 \mathrm{kDa}$ polypeptide at these same time points (Figure 5). No detectable cleavage of Bid was observed in cells transfected with mismatch siRNAs (Figure 5).

\section{Apo2L/TRAIL-induced cleavage of caspase-3 and PARP in melanoma cells transfected with siRNAs against IAPs}

Apo2L/TRAIL-induced caspase-3 cleavage was confirmed in melanoma cells by immunoblot analyses. Cells transfected with either control siRNA (si-Scr) or gene-specific siRNAs were treated with TNF- $\alpha$ or Apo2L/TRAIL for $8 \mathrm{~h}$ (maximum activation of caspase-3 was observed at 6-8h) and were immunoblotted with caspase-3 and PARP antibodies. Consistent with the fluorogenic substrate caspase activity assays, the $32 \mathrm{kDa}$ procaspase- 3 was cleaved to a $20 \mathrm{kDa}$ active form after Apo2L/TRAIL treatment of both untransfected or cells transfected with siRNAs. However, the p17 and p11 subunits resulting from the autocatalytic cleavage of caspase-3 were observed only in cells where expression of any one of the inhibitors was downregulated. No significant cleavage of caspase- 3 was observed following TNF- $\alpha$ in cells transfected with either si-Scr or si-IAPs (Figure 6a).

Furthermore, despite increased caspase-3 activity, PARP was not cleaved in control siRNA (si-Scr)-transfected cells treated with Apo2L/TRAIL (Figure 6b). Similar to si-Scr, no cleavage of PARP was observed in lipofectamine-control, siBcl-2 MM- or si-XIAP MM-transfected cells (data not shown). Consistent with TUNEL and Annexin V positivity, Apo2L/ TRAIL treatment resulted in cleavage of PARP only in cells in 


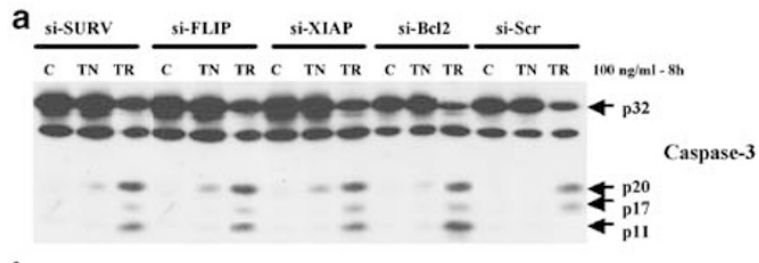

b

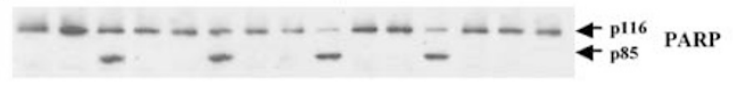

Figure 6 siRNA-IAPs and Apo2L/ TRAIL induced cleavage of caspase-3 and PARP in melanoma cells. siRNA-transfected A375 cells treated with TNF- $\alpha$ and TRAIL/Apo2L were washed twice with cold PBS and lysed on ice in cold lysis buffer. Cell lysates were subjected to Western blot analysis using caspase-3 and PARP antibodies, followed by HRP-conjugated secondary antibody. Blots were developed using enhanced chemiluminescence system. Procaspase $3(32 \mathrm{kDa})$ was cleaved to active $20 \mathrm{kDa}$ protein following Apo2L/TRAIL treatment in A375 cells transfected with control si-RNA. However, si-IAP/ TRAIL resulted in autocatalytic cleavage resulting in active p17/p11 fragment of caspase-3. Cleavage of the DNA repair enzyme, PARP, to its inactive $85 \mathrm{kDa}$ fragment was observed only in cells transfected with siRNAs against IAPs. No PARP cleavage was observed in cells treated with control siRNA. TNF- $\alpha$ failed to induce cleavage of either caspase-3 or PARP in untransfected or siRNA-transfected cells

which any one of the inhibitor of apoptosis was downregulated. No PARP cleavage was observed with TNF- $\alpha$ treatment in either si-Scr- or si-IAP-transfected cells (Figure 6b).

\section{Discussion}

Modulating the expression of apoptotic regulators such as $\mathrm{Bcl}-2$ and IAPs is an attractive strategy for probing their role in enhancing tumor cell apoptosis and thus defining potential therapeutic strategies. ${ }^{1,4,10,12}$ Mammalian gene function has traditionally been determined by methods such as murine knockout models, introduction of transgenes and or gene targeting by antisense oligonucleotides, ${ }^{30}$ ribozymes $^{31}$ or dsRNA interference techniques. ${ }^{20}$ RNA interference (RNAi) is a post-transcriptional gene silencing mechanism that is triggered by dsRNA. ${ }^{20-22}$ Gene silencing can be elicited effectively by specific 21-base duplex RNAs, termed siRNA without invoking generic antiviral host defense mechanisms. $^{21,22}$ Thus to minimize nonspecific cytotoxicity sometimes associated with antisense oligonucleotides or ribozymes, ${ }^{30,31}$ small synthetic siRNAs were utilized in this study.

Cells transfected with IAP-specific siRNAs potently suppressed specific target gene expression. At concentrations of siRNA used in this study $(20 \mathrm{nM})$, no off-target effects were observed. Si-XIAP and si-Bcl-2 downregulated XIAP and Bcl2 proteins, respectively, without effecting expression of other closely related genes such as survivin, Bax or Bad (Figure $1 \mathrm{~b}, \mathrm{c})$. Lipid-based transfection reagents induced a small increase in basal apoptosis (10-15\%) compared to untreated cells. No further increase in apoptosis associated with siRNA was observed. Since in some cells, siRNAs have been reported to induce IFN response, ${ }^{24,25}$ through activation of
dsRNA-activated protein kinase (PKR) and RNase $L$ pathway. ${ }^{25,32}$ To confirm the specificity of siRNAs used in this study, the IFN response in A375 melanoma cells was assessed following transfection with siRNAs. However, A375 cells transfected with IAP-specific siRNAs showed no induction of IFN-stimulated genes STAT-1 and ISG15 (Figure 1d).

Resistance to Apo2L/TRAIL has been attributed to differential expression of death receptors, ${ }^{33}$ defects in caspase$8,{ }^{34}$ higher expression of FLIP ${ }^{9}$ or XIAP ${ }^{19}$ or defects in Apaf$1^{35}$ expression. Apo2L/TRAIL binds to its receptors, activates caspase- 8 and results in cleavage of procaspase- 3 to active caspase-3 (p20/p12), yet does not induce apoptosis in melanoma cells. ${ }^{17}$ Tumors such as melanoma, glioma, prostate, colon and breast carcinoma, reported to be Apo2L/ TRAIL-resistant, have been rendered Apo2L/TRAIL sensitive by cotreatment with chemotherapeutic agents such as nitrosylcobalamin, ${ }^{27}$ actinomycin D (actD), irinotecan (CPT11), 5-fluorouracil ${ }^{36-39}$ and IFN- $\beta .^{17}$ These may inhibit expression of downstream inhibitory proteins like FLIP and IAPs that bind to FADD or other proteins in the caspase pathway. ${ }^{17-19}$

The $\mathrm{Bcl}-2$ gene is the prototype of a class of genes that contributes to neoplastic progression by enhancing tumor cell survival through the inhibition of apoptosis. ${ }^{6,7} \mathrm{Bcl}-2$ levels are elevated in follicular lymphoma, melanomas, prostate and gliomas, ${ }^{6,40}$ suggesting that a decrease in $\mathrm{Bcl}-2$ might sensitize cells to apoptotic stimuli. ${ }^{6,7,41}$ As hypothesized, downregulation of $\mathrm{Bcl}-2$ by siRNA sensitized melanoma cells to Apo2L/TRAIL-induced apoptosis (Figures 2,3). Apo2L/ TRAIL stimulated cleavage of Bid, caspase 3 (p17/p11) and PARP following downregulation of Bcl-2 in A375 melanoma cells (Figures 5,6). Thus, downregulation of $\mathrm{Bcl}-2$ may activate the mitochondrial arm (intrinsic pathway) to amplify the effects of death receptor-mediated apoptosis. ${ }^{42}$

Unlike Bcl-2 family members that elicit their antiapoptotic effects by regulating the intrinsic pathway, FLIP and other IAPs act predominantly by directly binding and inhibiting initiator as well as executioner caspases. FLIP (a dominant negative homolog of caspase-8) interacts with the adaptor protein FADD and caspase-8 (FLICE). FLIP forms heterodimers with caspase- 8 that are nonfunctional. These heterodimers are more stable than the functional caspase- 8 homodimers, resulting in inhibition of caspase-8 activity in cells overexpressing FLIP. ${ }^{8,9}$ In melanoma cells, Apo2L/ TRAIL resulted in increased activity of caspase-8 (2-2.5-fold) and caspase-3 (3-6-fold) but no apoptosis (Figure 4a,b). Thus, FLIP expression did not seem to have a direct role in mediating Apo2L/TRAIL resistance in melanomas.

Based on this observation, it was postulated that resistance to apoptosis lies downstream from caspase-8. However, a 26 -fold amplification of caspase- 8 and caspase- 3 activity and TUNEL positivity occurred following Apo2L/TRAIL treatment in A375 cells transfected with si-FLIP (Figure 2,4). In comparison with other targeted modulators of apoptosis, downregulation of FLIP resulted in a greater relative increase in caspase-8 activity. Caspase-8 itself cannot induce secondary cleavage of caspase-3, but it results in cleavage of Bid, affecting mitochondrial potential and in turn activating the intrinsic pathway. ${ }^{42,43}$ Increased caspase-8 activity may 
override the inhibitory effects of XIAP by disrupting the XIAPcaspase-3 (p20) interaction. ${ }^{43}$

$\mathrm{XIAP}$ is a most potent caspase inhibitor, ${ }^{12,29}$ directly binding and inhibiting caspase- 3 , caspase- 9 and caspase-7 activities. ${ }^{29}$ Infection of non-small-cell lung carcinoma or ovarian carcinoma cells with adenovirus expressing XIAP resulted in increased resistance to $\gamma$-irradiation- ${ }^{44}$ and cisplatin-induced apoptosis. ${ }^{45}$ Similarly, expression of survivin in tumor cells conferred a poor prognosis. ${ }^{46,47}$ Like other IAPs, survivin mediates its inhibitory function by inhibiting caspase- $3 .{ }^{10,46,47}$ As postulated, downregulation of either XIAP or survivin utilizing siRNAs sensitized tumor cells to apoptosis. Active XIAP or survivin may bind to the p20 subunit of caspase-3, preventing the second catalytic cleavage that is necessary for its activation. ${ }^{11,29,47}$ Compared to XIAP, melanoma cells did not express high levels of survivin, implicating XIAP as a significant inhibitor of Apo2L/TRAIL-induced apoptosis. Amplification of the caspase cascade in si-XIAP-transfected cells correlated with cleavage of Bid (Figure 5), indicating crosstalk between the extrinsic and intrinsic pathway. ${ }^{2,6}$ Increase in cell death correlated with increased caspase- 9 , caspase-8 and caspase-3 activity.

Despite comparable activation and cleavage of caspases in melanoma cells by siRNAs, inhibition of XIAP was the most potent in sensitizing cells to Apo2L/TRAIL-induced apoptosis (Figures 2,4). Resistance to Apo2L/TRAIL or TNF- $\alpha$ induced apoptosis has been attributed to the induction of $N F-\kappa B$, a survival factor. ${ }^{48,27}$ Nitrosylcobalamin (a nitric oxide donor) and curcumin sensitized cells to Apo2L/TRAIL by inhibiting NF- $\kappa$ B activation. ${ }^{27,49}$ Enhanced apoptosis by downregulation of XIAP could partly be due to inhibition of NF- $\kappa \mathrm{B}$ activity. ${ }^{50,51}$ Overexpression of XIAP, a NF- $\kappa$ B-dependent member of IAP family lead to increased nuclear translocation of $p 65$ subunit of NF- $k B$ involving mitogen-activated protein kinase kinase (TAK1), via phosphorylation and sustained degradation of inhibitor $(1 \kappa \mathrm{B} \alpha) .^{51}$ Moreover, an in -vitro ubiquitination assay identified XIAP as an ubiquitin-protein ligase for caspase $-3 .{ }^{52}$ XIAP promoted ubiquitination and subsequent degradation of active caspase- 3 , but not of procaspase-3. Thus, downregulation of XIAP may lead to decreased ubiquitin-protein ligase and NF- $\kappa$ B activity, resulting in increased sensitivity to apoptotic stimuli. ${ }^{13,50-52}$

Resistance to Apo2L/TRAIL was thus mediated by inhibition of both intrinsic and extrinsic apoptotic pathways. Compared to Apo2L/TRAIL, TNF- $\alpha$ ( a more potent inducer of NF- $\kappa \mathrm{B}$ ), may induce other antiapoptotic genes such as Bcl$X_{L}$ and C-IAP-2, thus potentially counteracting the apoptotic signaling by TNF- $\alpha .{ }^{48,53}$ Overall, the results highlight the relative importance of IAPs in mediating resistance to apoptosis and thus as potential targets for therapeutic interventions in cancer.

\section{Materials and Methods}

\section{Cell culture and IFN}

Melanoma (A375 (ATCC), WM9, WM35 (Dr. M. Herlyn, Wistar Institute), MUM2C, C918 (Dr. Mary Hendrix, University of lowa, lowa)) and renal cell carcinoma (SKRC45; Dr. Neil Bander, Cornell University, NY, USA) cell lines were grown in DMEM medium (Life Technologies Inc., Rockville,
MD,USA) supplemented with heat-inactivated $10 \%$ fetal calf serum (HyClone, Logan, UT, USA) in humidified chamber of $95 \%$ air $5 \% \mathrm{CO}_{2}$ at $37^{\circ} \mathrm{C}$. Apo2L/TRAIL (Genentech Inc., San Franscico, CA, USA) used in this study consisted of $>99 \%$ trimeric protein with $\mathrm{Zn}^{++} \cdot{ }^{28} \mathrm{Zn}^{++}$is necessary for optimal biologic activity.

\section{SIRNA}

SiRNAs against Bcl-2, XIAP, FLIP and survivin were synthesized by Dharmacon Inc. (Lafayette, CO, USA). All siRNAs were duplexed, desalted, $2^{\prime}$ deprotected and purified ( $>80 \%$ ) by Dharmacon Inc.

$\begin{array}{ll}\text { si-Bcl-2 MM: } & \text { combination of 5' AACAUCGCCCUGUGGAUGA- } \\ & \text { CU-3' and 5'AAGTACATCCAGTATCAGATG-3' } \\ \text { si-Bcl-2: } & \text { Dharmacon cat \# M-003307-00-05 } \\ \text { si-XIAP MM: } & \text { 5'AAGUCGUAGUGCUGUCUCACC3' } \\ \text { si- XIAP: } & \text { 5'AAGUGGUAGUCCUGUUUCAGC-3' }^{\prime} \\ \text { si-Flip: } & \text { 5'AACUGCUCUACAGAGUGAGGC-3' }^{\prime} \\ \text { si-Survivin: } & \text { 5'AAGGCUGGGAGCCAGAUGACG-3' }^{\prime} \text {-AAG } \\ \text { si-Scr: } & \text { Dharmacon cat \# D-001205-01-80 ( a non specific } \\ & \text { random scrambled sequence) }\end{array}$

\section{Transfection of SiRNA}

Cells were seeded at $10^{5} \mathrm{cells} / \mathrm{ml}$ in $10 \mathrm{~cm}$ dishes. Gene-specific siRNAs and the control siRNAs (20-40 nM) were added to media using lipophilic transfection reagent (Lipofectamine 2000, Invitrogen Inc., Carlsbad, CA, USA). Cells were lysed (48 and $72 \mathrm{~h}$ ) in $1 \times$ lysis buffer, ${ }^{17}$ incubated on ice for $20 \mathrm{~min}$, followed by centrifugation at $12000 \mathrm{~g}$ for $10 \mathrm{~min}$.

\section{Immunoblot analyses}

SDS PAGE was conducted by using Laemmli buffer system and $12 \%$ polyacrylamide gels. Whole cell lysates $(20 \mu \mathrm{g})$ were used for all immunoblotting experiments. Proteins separated on gels were transferred onto PVDF membrane by the semidry method (Trans Blot SD, BioRad, Hercules, CA, USA). Binding of the primary and secondary antibodies was performed in $1 \times$ Tris-buffered saline, pH 7.4 containing $5 \%(\mathrm{w} / \mathrm{v})$ nonfat dry milk and $0.2 \%(\mathrm{v} / \mathrm{v})$ Tween 20 for $1 \mathrm{~h}$ at room temperature. Membranes were immunoblotted with antibodies to Bcl-2, Bax, Bad, XIAP, FLIP, Bid (BD-Pharmingen, San Diego, CA, USA), survivin (Santacruz, Santacruz, CA, USA), caspase-3 and PARP (Biomol, Plymouth Meeting, PA, USA), followed by incubation with HRP-conjugated secondary antibodies (Pierce, Rockward, IL, USA). Immunoreactive bands were visualized by using enhanced chemiluminescence (Perkin Elmer, Boston, MA, USA).

\section{Quantitative real-time RT-PCR}

Primers for (UBE2L6, USP18 and GAPDH) were purchased from Applied Biosystems Inc. and used according to the manufacturer's instructions with Universal PCR Master Mix, No AmpErase UNG (Applied Biosystems Inc., Foster City, CA, USA). Briefly, A375 cells were transfected with lipofectamine 2000, si-Scr and si-XIAP. After $24 \mathrm{~h}$, RNA was isolated using TRIZOL (Invitrogen Inc., Carlsbad, CA, USA) following the manufacturer's protocol. RNA was converted to CDNA, and 200ng of CDNA was used with the Universal PCRMaster Mix on an ABI PRISM Sequence Detection Instrument 7700 . After 40 cycles, data reduction was performed with 
Sequence Detection System Software (Applied Biosystems Inc., Foster City, CA, USA).

\section{Apoptosis assays}

\section{TUNEL assay}

DNA fragmentation was assessed in siRNA and Apo2L/TRAlL-treated cells by TUNEL staining using the APO-BRDU ${ }^{\mathrm{TM}}$ kit (BD-Pharmingen, San Diego, CA, USA). Briefly, cells were washed with cold PBS, trypsinized and fixed in $1 \%$ paraformaldehyde for $15 \mathrm{~min}$ on ice. Fixed cells were washed twice with PBS, pelleted and suspended in 70\% ethanol. Cells were kept $>24 \mathrm{~h}$ at $-20^{\circ} \mathrm{C}$ in $70 \%$ ethanol. For labeling with bromolated deoxyribonucleotide triphosphates (Br-dUTP), cells were washed twice with PBS, labeled with Br-dUTP and the enzyme TdT for $1 \mathrm{~h}$ at $37^{\circ} \mathrm{C}$. After labeling, cells were washed and stained with FITCconjugated anti-BrdU mAb for $30 \mathrm{~min}$ in a low light environment. RNase-PI was added and the samples were incubated for an additional $30 \mathrm{~min}(\mathrm{RT})$. The percentage of FITC-positive cells was analyzed by fluorescentactivated cell scanning (FACS) (Becton Dickinson Facsvantage).

\section{Caspase activity assay}

Caspase-3, caspase-8 and caspase-9 activities were measured using a commercially available ApoAlert assay kit (Clonetech, Palo Alto, CA, USA). siRNA-transfected cells either left untreated or treated with TRAIL/ Apo2L (1, 2 and $4 \mathrm{~h}$ ) were washed twice with cold PBS and lysed on ice in $50 \mu \mathrm{l}$ of cold lysis buffer. Cell lysates were centrifuged at $10000 \times g$ for $10 \mathrm{~min}$ to precipitate cellular debris. Assay was performed in triplicates in a 96-well plate based on the manufacturer's protocol.

\section{Annexin V/PI assay}

Annexin V staining of exposed membrane phospholipid phosphatidylserine was carried out using the Annexin $V$ assay kit (BD-Pharmingen, San Diego, CA, USA) following the manufacturer's protocol. The percent Annexin $\mathrm{V}$ and PI-positive cells were analyzed by FACS.

\section{Acknowledgements}

We thank Dr. Avi Ashkenazi (Genentech, Inc., San Franscisco, CA, USA) for providing recombinant soluble Apo2L/TRAIL protein. We gratefully acknowledge the technical assistance by Alex Rodriguez for flow cytometry. This study was supported by NIH R01 Grant CA 90914 (EC Borden).

\section{References}

1. Reed JC (2002) Apoptosis-based therapies. Nat. Rev. Drug Discov. 1: 111121

2. Hengartner MO (2000) The biochemistry of apoptosis. Nature 407: 770-776

3. Green DR and Evan GI (2002) A matter of life and death. Cancer Cell 1: 19-30

4. LaCasse EC, Baird S, Korneluk RG and MacKenzie AE (1998) The inhibitors of apoptosis (IAPs) and their emerging role in cancer. Oncogene 17: 3247-3259

5. Green DR and Reed JC (1998) Mitochondria and apoptosis. Science 28: 13091312

6. Gross A, McDonell JM and Korsmeyer SJ (1999) Bcl-2 family members and mitochondria in apoptosis. Genes Dev. 13: 1899-1911

7. Hockenbery D, Nunez G, Milliman C, Schreiber RD and Korsmeyer SJ (1990) $\mathrm{Bcl}-2$ is an inner mitochondrial membrane protein that blocks programmed cell death. Nature 348: 334-336
8. Hu S, Vincenz C, Ni J, Gentz R and Dixit VM (1997) I-FLICE, a novel inhibitor of tumor necrosis factor receptor-1 and CD-95-induced apoptosis. J. Biol. Chem. 272: $17255-17257$

9. Irmler M, Thome M, Hahne M, Schneider P, Hofmann K, Steiner V, Bodmer JL, Schroter M, Burns K, Mattmann C, Rimoldi D, French LE and Tschopp J (1997) Inhibition of death receptor signals by cellular FLIP. Nature 388: 190-195

10. Altieri DC (2003) Validating survivin as a cancer therapeutic target. Nat. Rev. Cancer 3: 46-54

11. Deveraux QL and Reed JC (1999) IAP family proteins-suppressors of apoptosis. Genes Dev. 13: 239-252

12. Salvesen GS and Duckett CS (2002) IAP proteins: blocking the road to death's door. Nat. Rev. Mol. Cell Biol. 3: 401-410

13. Yang Y, Fang S, Jensen JP, Weissman AM and Ashwell JD (2000) Ubiquitin protein ligase activity of IAPs and their degradation in proteasomes in response to apoptotic stimuli. Science 288: 874-877

14. Wiley SR, Schooley K, Smolak PJ, Din WS, Huang CP, Nicholl JK, Sutherland GR, Smith TD, Rauch C, Smith CA and Goodwin RG (1995) Identification and characterization of a new member of the TNF family that induces apoptosis. Immunity 3: 673-682

15. Pitti RM, Marsters SA, Ruppert S, Donahue CJ, Moore A and Ashkenazi A (1996) Induction of apoptosis by Apo-2 ligand, a new member of the tumor necrosis factor cytokine family. J. Biol. Chem. 271: 12687-12690

16. Chawla-Sarkar M, Leaman DW and Borden EC (2001) Preferential induction of apoptosis by interferon (IFN)- $\beta$ compared with IFN- $\alpha 2$ : correlation with TRAIL/Apo2L induction in melanoma cell lines. Clin. Cancer Res. 7: 1821-1831

17. Chawla-Sarkar M, Leaman DW, Jacobs BS and Borden EC (2002) IFN-beta pretreatment sensitizes human melanoma cells to TRAIL/Apo2 ligand-induced apoptosis. J. Immunol. 169: 847-855

18. Dong Zhang X, Franco AV, Myers K, Gray CP, Nguyen T and Hersey P (1999) Relation of TNF-related apoptosis inducing ligand (TRAIL) receptor and Flice inhibitory protein expression to TRAIL induced apoptosis of melanoma cells. Cancer Res. 59: 2747-2753

19. Dong Zhang $X$, Zhang $X Y$, Gray CP, Nguyen T and Hersey P (2001) Tumor necrosis factor related apoptosis inducing ligand induced apoptosis of human melanoma is regulated by Smac/DIABLO release from mitochondria. Cancer Res. 61: 7339-7348

20. McManus MT and Sharp PA (2002) Gene silencing in mammals by small interfering RNAs. Nat. Rev. Genet. 3: 737-747

21. Chi JT, Chang HY, Wang NN, Chang DS, Dunphy N and Brown PO (2003) Genomewide view of gene silencing by small interfering RNAs. Proc. Natl. Acad. Sci. USA 100: 6343-6346

22. Gitlin L, Karelsky S and Andino R (2002) Short interfering RNA confers intracellular antiviral immunity in human cells. Nature 418: 430-434

23. Jackson AL, Bartz SR, Schelter J, Kobayashi SV, Burchard J, Mao M, Li B, Cavet $G$ and Linsley PS (2003) Expression profiling reveals off-target gene regulation by RNAi. Nat. Biotech. 21: 635-637

24. Bridge AJ, Pebernard S, Ducraux A, Nicoulaz AL and lggo R (2003) Induction of an interferon response by RNAi vectors in mammalian cells. Nat. Genet. 34: 263-264

25. Sledz CA, Holko M, de Veer MJ, Silverman RH and Williams BRG (2003) Activation of the interferon system by short interfering RNAs. Nat. Cell Biol. 5 : 834-839

26. de Veer MJ, Holko M, Frevel M, Walker E, Der S, Paranjpe JM, Silverman RH and Williams BR (2001) Functional classification of interferon-stimulated genes identified using microarrays. J. Leukoc. Biol. 69: 912-920

27. Chawla-Sarkar M, Bauer JA, Lupica JA, Morrison BH, Tang Z, Oates RK, Almasan A, DiDonato JA, Borden EC and Lindner DJ (2003) Suppression of $\mathrm{NF}-\kappa \mathrm{B}$ survival signaling by nitrosylcobolomin sensitizes neoplasms to antitumor effects of Apo2L/TRAIL. J. Biol. Chem. 278: 39461-39469

28. Ashkenazi A, Pai RC, Fong S, Leung S, Lawrence DA, Marsters SA, Blackie C, Chang L, McMurtrey AE, Hebert A, DeForge L, Koumenis IL, Lewis D, Harris L, Bussiere J, Koeppen H, Shahrokh Z and Schwall RH (1999) Safety and antitumor activity of recombinant soluble Apo2 ligand. J. Clin. Invest. 104: 155162

29. Deveraux QL, Takahashi R, Salvesan GS and Reed JC (1997) X-linked IAP is a direct inhibitor of cell-death proteases. Nature 388: 300-304

30. Kushner DM and Silverman RH (2000) Antisense cancer therapy: the state of the science. Curr. Oncol. Rep. 2: 23-30 
31. Usman N and Blatt LM (2000) Nuclease-resistant synthetic ribozymes: developing a new class of therapeutics. J. Clin. Invest. 106: 1197-1202

32. Williams BR (1999) PKR; a sentinel kinase for cellular stress. Oncogene 18 $6112-6120$

33. Griffith TS, Chin WA, Jackson GC, Lynch DH and Kubin MZ (1998) Intracellular regulation of TRAIL-induced apoptosis in human melanoma cells. J. Immunol. 161: $2833-2840$

34. Eggert A, Grotzer MA, Zuzak TJ, Wiewrodt BR, Ho R, Ikegaki N and Brodeur GM (2001) Resistance to tumor necrosis factor related apoptosis inducing ligand-induced apoptosis in neuroblastoma cells correlates with loss of caspase-8 expression. Cancer Res. 61: $1314-1319$

35. Soengas MS, Capodleci P, Polsky D, Mora J, Esteller M, Opitz-Araya X, McCombie R, Herman JG, Gerald WL, Lazebnik YA, Cardo-Cordon C and Lowe SW (2001) Inactivation of apoptosis effector Apaf-1 in malignant melanoma. Nature 409: 207-211

36. Gliniak B and Le T (1999) Tumor necrosis factor related apoptosis inducing ligand's antitumor activity in vivo is enhanced by the chemotherapeutic agent CPT11. Cancer Res. 59: 6153-6158

37. Keane MM, Ettenberg SA, Nau MM, Russell EK and Lipkowitz S (1999) Chemotherapy augments TRAIL-induced apoptosis in breast cancer cell lines. Cancer Res. 59: 734-741

38. Zisman A, Ng CP, Pantuck AJ, Bonavida B and Belldegrun AS (2001) Actinomycin $D$ and gemcitabine synergistically sensitize androgen independent prostrate cancer cells to Apo2L/TRAIL mediated apoptosis. J. Immunother 6 : $459-471$

39. Mizutani Y, Nakanishi H, Yoshida O, Fukushima M, Bonavida B and Miki T (2002) Potentiation of the sensitivity of renal cell carcinoma cells to TRAILmediated apoptosis by subtoxic concentrations of 5 -Fluorouracil. Eur. J. Cancer 38: $167-176$

40. Weisenburger DD, Gascoyne RD, Bierman PJ, Shenkier T, Horsman DE, Lynch JC, Chan WC, Greiner TC, Connors JM, Vose JM, Armitage JO and Sanger WG (2000) Clinical significance of the $t(14 ; 18)$ and $B C L 2$ overexpression in follicular large cell lymphoma. Leuk. Lymphoma 36: 513-523

41. Jansen B and Zangemeister-Wittke $U$ (2002) Antisense therapy for cancer the time of truth. Lancet Oncol. 3: 672-683 Erratum in: Lancet Oncol. 2003 4: 74.
42. Kuwana T, Smith JJ, Muzio M, Dixit V, Newmeyer DD and Kornbluth S (1998) Apoptosis induction by caspase-8 is amplified through the mitochondrial release of cytochrome c. J. Biol. Chem. 273: 16589-16594

43. Siegmund D, Hadwiger P, Pfizenmaier K, Vornlocher HP and Wajant $H$ (2002) Selective inhibition of FLICE-like inhibitory protein expression with small interfering RNA oligonucleotides is sufficient to sensitize tumor cells for TRAILinduced apoptosis. Mol. Med. 8: 725-732

44. Holcik M, Yeh C, Korneluk RG and Chow T (2000) Translational upregulation of $X$-linked inhibitor of apoptosis (XIAP) increases resistance to radiation induced cell death. Oncogene 19: 4174-4177

45. Sasaki H, Sheng Y, Kotsuji F and Tsang BK (2000) Down-regulation of X-linked inhibitor of apoptosis protein induces apoptosis in chemoresistant human ovarian cancer cells. Cancer Res. 60: 5659-5666

46. Ambrosini G, Adida C and Altieri DC (1997) A novel anti-apoptosis gene, survivin, expressed in cancer and lymphoma. Nat. Med. 8: 917-921

47. Ambrosini G, Adida C, Sirugo G and Altieri DC (1998) Induction of apoptosis and inhibition of cell proliferation by survivin gene targeting. J. Biol. Chem. 273: $11177-11182$

48. Gupta S (2002) A decision between life and death during TNF-alpha-induced signaling. J. Clin. Immunol. 22: 185-194

49. Deeb D, Xu YX, Jiang H, Gao X, Janakiraman N, Chapman RA and Gautam SC (2003) Curcumin (diferuloyl-methane) enhances tumor necrosis factorrelated apoptosis-inducing ligand-induced apoptosis in LNCaP prostate cancer cells. Mol. Cancer Ther. 2: 95-103

50. Levkau B, Garton KJ, Ferri N, Kloke K, Nofer J-R, Baba HA, Raines EW and Breithardt G (2001) XIAP induces cell-cycle arrest and activates nuclear factor$\mathrm{kB}$ : new survival pathways disabled by caspase mediated cleavage during apoptosis of human endothelial cells. Circ. Res. 88: 282-290

51. Hofer-Warbinek R, Schmid JA, Stehlik C, Binder BR, Lipp J and Martin R (2000) Activation of NF-kB by XIAP, the X chromosome-linked inhibitor of apoptosis, in endothelial cells involves TAK1. J. Biol. Chem. 275: 22064-22068

52. Suzuki $Y$, Nakabayashi $Y$ and Takahashi $R$ (2001) Ubiquitin-protein ligase activity of $X$-linked inhibitor of apoptosis protein promotes proteasomal degradation of caspase-3 and enhances its anti-apoptotic effect in Fas-induced cell death. Proc. Natl. Acad. Sci. USA 98: 8662-8667

53. Wang CY, Mayo MW, Korneluk RG, Goeddel DV and Baldwin Jr AS (1998) NFkappaB antiapoptosis: induction of TRAF1 and TRAF2 and C-IAP1 and c-IAP2 to suppress caspase-8 activation. Science 281: 1680-1683 